Acta Crystallographica Section D

Biological

Crystallography

ISSN 0907-4449

\section{Fabrice Gorrec, ${ }^{*}$ Colin M. Palmer, Guillaume Lebon and Tony Warne}

MRC Laboratory of Molecular Biology, Hills Road, Cambridge CB2 OQH, England

Correspondence e-mail: fgorrec@mrc-Imb.cam.ac.uk

\title{
Pi sampling: a methodical and flexible approach to initial macromolecular crystallization screening
}

The $\mathrm{Pi}$ sampling method is derived from the incomplete factorial approach to macromolecular crystallization screen design. The resulting 'Pi screens' have a modular distribution of a given set of up to 36 stock solutions. Maximally diverse conditions can be produced by taking into account the properties of the chemicals used in the formulation and the concentrations of the corresponding solutions. The Pi sampling method has been implemented in a web-based application that generates screen formulations and recipes. It is particularly adapted to screens consisting of 96 different conditions. The flexibility and efficiency of Pi sampling is demonstrated by the crystallization of soluble proteins and of an integral membrane-protein sample.

\section{Introduction}

A crucial aspect of macromolecular crystallographic studies is finding suitable conditions for the crystallization of a sample. This can be difficult because many factors alter the crystallization behaviour of macromolecules, including the type and the concentration of the chemicals employed to formulate the conditions (McPherson, 1990). A condition includes at least a precipitant and most conditions also include a buffer and an additive. During the initial crystallization experiments, the structure of the macromolecule is not known and hence the most efficient formulation cannot be predicted. As a consequence, one should be cautious when making initial assumptions and limiting choices in subsequent optimizations (Rupp, 2003). Nonetheless, the number of initial crystallization conditions cannot be unreasonably large since purified protein is often difficult and expensive to produce in large quantities.

There are essentially two approaches to restrict an initial screen to a limited number of crystallization conditions. Firstly, a sparse-matrix formulation can be used, which consists of an empirically derived combination of components based on known or published crystallization conditions (Jancarik \& Kim, 1991). Secondly, an incomplete factorial formulation can be generated in which selected components are combined to prepare new conditions in accordance with principles of randomization and balance (Carter \& Carter, 1979). Numerous commercial screens based on these two main approaches are available. Automated systems have been implemented at the Medical Research Council (MRC) Laboratory of Molecular Biology (LMB) to test these as
Received 4 January 2011

Accepted 7 March 2011 
routine initial screens using the 96-well crystallization plate format (Stock et al., 2005). However, for various reasons, many laboratories opt for a minimal screen (Kimber et al., 2003) and still perform at least some aspects of the work manually (Bergfors, 2007).

Here, we present a development based on the incomplete factorial formulation: the Pi sampling method. The name of the method was inspired by the story of Archimedes, who used the 'method of exhaustion' (i.e. an empirical approach) with a 96-sided polygon in order to reach the first good numerical approximation of $\pi$ (Smith, 1958). Pi sampling uses modular arithmetic to form combinations of three stock solutions across a 96-condition grid. Maximally diverse conditions can

\begin{tabular}{|c|c|c|c|c|c|c|c|c|c|c|c|c|}
\cline { 2 - 14 } \multicolumn{1}{c|}{} & M & N & O & P & Q & R & S & T & U & V & W & X \\
\hline A & 1 & 12 & 11 & & 9 & & 7 & 6 & & 4 & & 2 \\
\hline B & 3 & 2 & 1 & 12 & & 10 & & 8 & 7 & & 5 & \\
\hline C & & 4 & 3 & 2 & 1 & & 11 & & 9 & 8 & & 6 \\
\hline D & 7 & & 5 & 4 & 3 & 2 & & 12 & & 10 & 9 & \\
\hline E & & 8 & & 6 & 5 & 4 & 3 & & 1 & & 11 & 10 \\
\hline F & 11 & & 9 & & 7 & 6 & 5 & 4 & & 2 & & 12 \\
\hline G & 1 & 12 & & 10 & & 8 & 7 & 6 & 5 & & 3 & \\
\hline H & & 2 & 1 & & 11 & & 9 & 8 & 7 & 6 & & 4 \\
\hline I & 5 & & 3 & 2 & & 12 & & 10 & 9 & 8 & 7 & \\
\hline J & & 6 & & 4 & 3 & & 1 & & 11 & 10 & 9 & 8 \\
\hline K & 9 & & 7 & & 5 & 4 & & 2 & & 12 & 11 & 10 \\
\hline L & 11 & 10 & & 8 & & 6 & 5 & & 3 & & 1 & 12 \\
\hline
\end{tabular}

Figure 1

Pi sampling: combinations of stock solutions from three different sets (see also http://pisampler.mrc-lmb.cam.ac.uk/). be produced by taking into account the properties of the chemicals used in the formulation and the concentrations of the corresponding stock solutions. We have implemented this approach in a web-based application called Pi Sampler: user input consists of the details of up to 36 stock solutions, from which the application generates the formulations for a 96-condition screen. The Pi sampling method is intended to help laboratories to test new crystallization-screen formulations on a day-to-day basis based on the properties of the macromolecules investigated, as has been performed previously with RNA (Doudna et al., 1993).

Firstly, we tested Pi sampling with ten commercially available soluble proteins. For this, the 'Pi minimal screen' was employed including a wide variety of well known chemicals frequently used for macromolecular crystallization.

We then investigated the impact of Pi sampling on the crystallization of a G-protein-coupled receptor (GPCR) that had been difficult to crystallize: the adenosine $A_{2 A}$ receptor (construct $\mathrm{A}_{2 \mathrm{~A}} \mathrm{R}-\mathrm{GL} 31$ ). We formulated another Pi screen, the 'Pi-PEG screen', taking into consideration general observations made about crystallization of integral membrane-protein samples. Previous crystallization experiments on another GPCR (the $\beta_{1}$-adrenergic receptor) had indicated that the use of simple proprietary screens formulated with poly(ethylene glycol) (PEG) and buffers gave a greater yield of crystals than all commercially available screens, including those geared towards membrane proteins (Warne et al., 2009), and the $2.7 \AA$ resolution structure was solved using conditions optimized from a proprietary screen essentially based on PEGs (Warne et al., 2008). This has been observed previously with other membrane-protein targets (Lemieux et al., 2003). In addition, mixtures of polyethylene glycols have been used successfully to develop a minimal screen (Brzozowski \& Walton, 2001) and to study crystal structures of the Kir potassium channel (Clarke et al., 2010). Such mixtures were incorporated into the Pi-PEG screen.

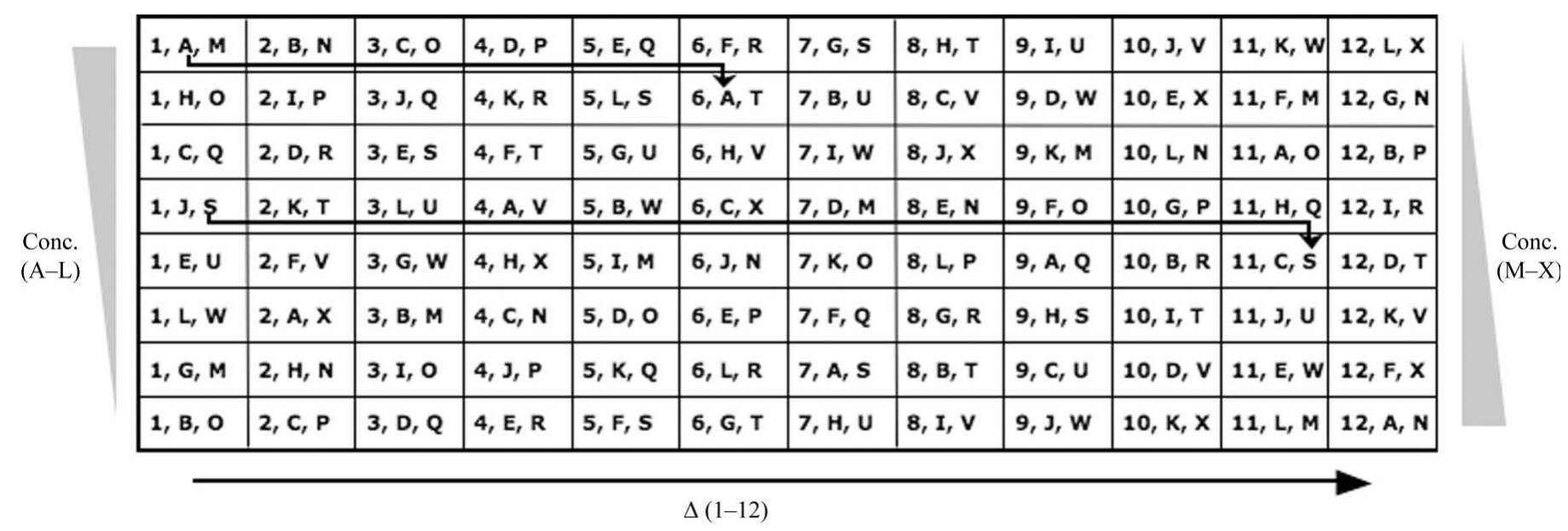

\section{Figure 2}

Pi sampling: combinations of the stock solutions in a 96-condition plate layout (well A1 is at the top left corner). Each solution of set 1 (ID 1-12) is seen in the eight conditions forming a column of the plate. The $\Delta$ values of set 1 increase from left to right in the screen layout. The positions of the solutions A-L (set 2) shift across five columns and down one row ( $\Delta$ values not represented). The positions of solutions M-X (set 3 ) shift across ten columns and down one row. Gradients of concentration for sets 2 and 3 are represented on the left and right, respectively. 


\section{Methods}

\subsection{Pi sampling}

Pi sampling begins with up to 36 stock solutions, divided into three sets of 12 . The first set of solutions is used in the screen at constant concentration. The second and third sets are added according to a gradient between specified minimum and maximum concentrations. Typically, the first set is composed of buffers and the second and third sets are precipitants/ additives.

The combinations of three stock solutions (one from each set) are generated according to Fig. 1, where 1-12 refer to the IDs for solutions of the first set, A-M to those of the second set and $\mathrm{N}-\mathrm{X}$ to those of the third set. The number in each cell shows which solution of the first set will be combined with the corresponding solutions of the second and third sets. Blank spaces show when no such combinations are generated.

Fig. 2 summarizes the distribution of the stock solutions in a standard 96-condition plate layout (i.e. 12 columns and eight rows).

Set 1: each solution (ID 1-12) is seen in the eight conditions forming a column of the plate. A variable $\Delta$ should be associated with the stock solutions. The variable $\Delta$ corresponds to a property of the solution selected (e.g. $\mathrm{pH}$, molecular weight of the main chemical, absorption properties or others). $\Delta$ values increase from left to right in the screen layout.

Set 2: each solution (ID A-L) is represented once in each row. The final concentrations decrease gradually from the top to the bottom of the screen layout, forming a gradient. The distribution of solutions $\mathrm{A}-\mathrm{L}$ is based on the sequence of $\Delta$ values established for set 1 : the positions of the solutions shift across five columns and down one row. Solutions A-L should also be associated with a variable $\Delta$ and hence a sequence is formed for the distribution of the third set of solutions.

Set 3: each solution (ID M-X) is also represented once in each row. The final concentrations increase gradually from the top to the bottom of the screen, forming another gradient. The solutions $\mathrm{M}-\mathrm{X}$ are distributed with the same modulo arithmetic operation as previously, but with respect to the $\Delta$ values of solutions A-L. For example, solution $\mathrm{M}$ is mixed with solution $\mathrm{A}$ in the first row, solution $\mathrm{F}$ in the second row, solution $\mathrm{K}$ in the third row and so on, as shown in Fig. 2. This means that both the second and third sets are arranged according to the same modulo arithmetic operation (5 modulo 12); however, when looking at the plate layout, the positions of solutions $\mathrm{M}-\mathrm{X}$ shift across ten columns and down one row.

\subsection{Pi Sampler}

$P i$ Sampler can be accessed via the internet at http:// pisampler.mrc-lmb.cam.ac.uk/. Users can enter the details of up to 36 stock solutions, including stock concentrations, desired screen concentration ranges and $\Delta$ values. The application then generates a 96-condition screen formulation following the Pi sampling method described above. Formulations, recipes and total required volumes of stock solutions are presented and may conveniently be downloaded in comma- separated variable format (CSV), allowing the user to import them into other software for automated screen making (Cox \& Weber, 1987), formulation analysis (Hedderich et al., 2011) and data mining (Kantardjieff \& Rupp, 2004). The parameters used to generate the screen can also be saved and uploaded in the same format. Further details and instructions can be found on the website.

\subsection{Pi minimal screen preparation and crystallization assays with commercially available soluble proteins}

The final formulation of the Pi minimal screen can be found in Table 1. There are 36 starting stock solutions overall. Each solution composing the first set (ID 1-12) is a mixture of an acid with its corresponding base (e.g. HEPES pH 7.5: $1 \mathrm{M}$ HEPES solution mixed with $1 M$ HEPES sodium salt in order to reach $\mathrm{pH} 7.5$ ), except for buffer 11 (AMPD mixed with Tris base). Note that this is also true for the precipitant phosphate (phosphate system: sodium dihydrogen phosphate/dipotassium hydrogen phosphate). Values of $\mathrm{pH}$ (4.0-9.5) were chosen as the variable $\Delta$ for the first set, whilst arbitrary values were chosen for additives of various natures composing the second set (ID A-L). Eventually, a few conditions were made without additive/buffer because of chemical incompatibilities (Table 1).

Highest purity grade chemicals (Molecular Biology grade when available) were purchased from Sigma-Aldrich to prepare 36 stock solutions. The solutions were mixed in 96 Falcon tubes. The screen was dispensed into 'MRC original plates' (96-well, two-drop, Swissci; Stock et al., 2005).

Commercial proteins that had been crystallized before were chosen to prepare test samples. Protein concentrations were chosen randomly between 7 and $150 \mathrm{mg} \mathrm{ml}^{-1}$ (Table 2). Vapour-diffusion experiments were set up at $295 \mathrm{~K}$, mixing two different sample: condition ratios (1:3 and 3:1) to give a final volume of $400 \mathrm{nl}$. The plates were then stored at $291 \mathrm{~K}$. A condition was considered to be a hit when at least one of the two corresponding drops contained crystals with well known morphology after one week. Table 3 shows the 'hits per condition' observed and the corresponding results expected for the binomial distribution (see $\$ 4.2$ ).

\subsection{Pi-PEG screen preparation and crystallization assays with a GPCR}

The final formulation of the Pi-PEG screen can be found in Table 4. The formulation can also be generated using $P i$ Sampler by loading the Pi-PEG example data. The $\mathrm{pH}$ values (4.8-8.8) were chosen as the variable $\Delta$ for the buffers composing set 1 (ID 1-12), whilst molecular weight was chosen for set 2 (PEGs A-L, final concentration range 0$22.5 \%$ ). The same 12 PEGs were used for set 3 (PEGs M-X, final concentration range $0-45 \%)$. General details of the preparation are similar to $\$ 2.3$, but there are 24 stock solutions at the start (instead of 36). Vapour-diffusion experiments were set up at $277 \mathrm{~K}$, mixing sample and condition in a 1:1 ratio to give a final volume of $200 \mathrm{nl}$. The preparation of $\mathrm{A}_{2 \mathrm{~A}} \mathrm{R}$-GL31 will be published elsewhere (Lebon et al., submitted work). Crystal X-ray screening was performed at the Diamond 
Table 1

Final formulation of the Pi minimal screen.

ADA, $N$-(2-acetamido)iminodiacetic acid; AMPD, 2-amino-2-methyl-1,3-propanediol; CAPSO, 3-(cyclohexylamino)-2-hydroxy-1-propanesulfonic acid; HEPES, 4-(2-hydroxyethyl)piperazine-1-ethanesulfonic acid; MOPS, 3-( $N$-morpholino)propanesulfonic acid; PEG, poly(ethylene glycol); TAPS, $N$-[Tris(hydroxymethyl)methyl]-3-aminopropanesulfonic acid.

\begin{tabular}{|c|c|c|c|c|c|c|c|c|c|c|c|c|}
\hline \multirow[b]{2}{*}{ Well } & \multicolumn{4}{|c|}{ Set 1} & \multicolumn{4}{|c|}{ Set 2} & \multicolumn{4}{|c|}{ Set 3} \\
\hline & ID & Name & Conc. & Unit & ID & Name & Conc. & Unit & ID & Name & Conc. & Unit \\
\hline A1 & 1 & Formate $\mathrm{pH} 4.0$ & 0.15 & $M$ & A & Potassium bromide & 0.160 & $M$ & M & Phosphate & 0.6 & $M$ \\
\hline A2 & 2 & Acetate $\mathrm{pH} 4.5$ & 0.15 & $M$ & B & PEG 300 & 8.000 & $\%(v / v)$ & $\mathrm{N}$ & PEG MME 550 & 24.00 & $\%(v / v)$ \\
\hline A3 & 3 & Malate pH 5.0 & 0.15 & $M$ & $\mathrm{C}$ & Magnesium sulfate & 0.160 & $M$ & $\mathrm{O}$ & Ammonium nitrate & 2.0 & $M$ \\
\hline A4 & 4 & Citrate $\mathrm{pH} 5.5$ & 0.15 & $M$ & $\mathrm{D}$ & Sodium fluoride & 0.032 & $M$ & $\mathrm{P}$ & PEG 20000 & 10.0 & $\%(w / v)$ \\
\hline A5 & 5 & MES pH 6.0 & 0.15 & $M$ & $\mathrm{E}$ & Potassium thiocyanate & 0.080 & $M$ & $\mathrm{Q}$ & PEG 1000 & 30.0 & $\%(w / v)$ \\
\hline A6 & 6 & Cacodylate pH 6.5 & 0.15 & $M$ & $\mathrm{~F}$ & Sodium iodide & 0.160 & $M$ & $\mathrm{R}$ & Sodium chloride & 1.6 & $M$ \\
\hline A7 & 7 & MOPS pH 7.0 & 0.15 & $M$ & G & Propanediol & 8.000 & $\%(v / v)$ & $\mathrm{S}$ & PEG 4000 & 24.0 & $\%(w / v)$ \\
\hline A8 & 8 & HEPES pH 7.5 & 0.15 & $M$ & $\mathrm{H}$ & & & & $\mathrm{T}$ & Lithium sulfate & 0.8 & $M$ \\
\hline A9 & 9 & Tris pH 8.0 & 0.15 & $M$ & I & Ethylene glycol & 8.000 & $\%(v / v)$ & $\mathrm{U}$ & PEG MME 5000 & 20.0 & $\%(w / v)$ \\
\hline A10 & 10 & TAPS pH 8.5 & 0.15 & $M$ & $\mathrm{~J}$ & Sodium potassium tartrate & 0.080 & $M$ & $\mathrm{~V}$ & Glycerol & 36.0 & $\%(w / v)$ \\
\hline A11 & 11 & AMPD/Tris pH 9.0 & 0.15 & $M$ & $\mathrm{~K}$ & MPD & 8.000 & $\%(v / v)$ & W & Ammonium sulfate & 1.4 & M \\
\hline A12 & 12 & CAPSO pH 9.5 & 0.15 & $M$ & $\mathrm{~L}$ & 2-Butanol & 8.000 & $\%(v / v)$ & $\mathrm{X}$ & PEG 8000 & 20.0 & $\%(w / v)$ \\
\hline B1 & 1 & Formate $\mathrm{pH} 4.0$ & 0.15 & $M$ & $\mathrm{H}$ & Calcium chloride & 0.070 & $M$ & $\mathrm{O}$ & Ammonium nitrate & 2.3 & M \\
\hline B2 & 2 & Acetate $\mathrm{pH} 4.5$ & 0.15 & $M$ & I & Ethylene glycol & 7.000 & $\%(v / v)$ & $\mathrm{P}$ & PEG 20000 & 12.0 & $\%(w / v)$ \\
\hline B3 & 3 & Malate pH 5.0 & 0.15 & $M$ & $\mathrm{~J}$ & Sodium potassium tartrate & 0.070 & $M$ & Q & PEG 1000 & 35.0 & $\%(w / v)$ \\
\hline B4 & 4 & Citrate pH 5.5 & 0.15 & $M$ & $\mathrm{~K}$ & MPD & 7.000 & $\%(v / v)$ & $\mathrm{R}$ & Sodium chloride & 1.8 & M \\
\hline B5 & 5 & MES pH 6.0 & 0.15 & $M$ & $\mathrm{~L}$ & 2-Butanol & 7.000 & $\%(v / v)$ & $\mathrm{S}$ & PEG 4000 & 28.0 & $\%(w / v)$ \\
\hline B6 & 6 & Cacodylate $\mathrm{pH} 6.5$ & 0.15 & $M$ & A & Potassium bromide & 0.140 & M & $\mathrm{T}$ & Lithium sulfate & 0.9 & $M$ \\
\hline B7 & 7 & MOPS pH 7.0 & 0.15 & $M$ & B & PEG 300 & 7.000 & $\%(v / v)$ & $\mathrm{U}$ & PEG MME 5000 & 23.0 & $\%(w / v)$ \\
\hline B8 & 8 & HEPES pH 7.5 & 0.15 & $M$ & $\mathrm{C}$ & Magnesium sulfate & 0.140 & $M$ & V & Glycerol & 42.0 & $\%(w / v)$ \\
\hline B9 & 9 & Tris pH 8.0 & 0.15 & $M$ & $\mathrm{D}$ & Sodium fluoride & 0.028 & $M$ & W & Ammonium sulfate & 1.6 & $M$ \\
\hline B10 & 10 & TAPS pH 8.5 & 0.15 & $M$ & E & Potassium thiocyanate & 0.070 & $M$ & $\mathrm{X}$ & PEG 8000 & 23.0 & $\%(w / v)$ \\
\hline B11 & 11 & AMPD/Tris pH 9.0 & 0.15 & $M$ & $\mathrm{~F}$ & Sodium iodide & 0.140 & $M$ & M & Phosphate & 0.7 & $M$ \\
\hline B12 & 12 & CAPSO pH 9.5 & 0.15 & $M$ & G & Propanediol & 7.000 & $\%(v / v)$ & $\mathrm{N}$ & PEG MME 550 & 28.00 & $\%(v / v)$ \\
\hline $\mathrm{C} 1$ & 1 & Formate $\mathrm{pH} 4.0$ & 0.15 & $M$ & $\mathrm{C}$ & Magnesium sulfate & 0.120 & $M$ & Q & PEG 1000 & 39.0 & $\%(w / v)$ \\
\hline $\mathrm{C} 2$ & 2 & Acetate $\mathrm{pH} 4.5$ & 0.15 & $M$ & $\mathrm{D}$ & Sodium fluoride & 0.024 & $M$ & $\mathrm{R}$ & Sodium chloride & 2.1 & $M$ \\
\hline $\mathrm{C} 3$ & 3 & Malate pH 5.0 & 0.15 & $M$ & $\mathrm{E}$ & Potassium thiocyanate & 0.060 & $M$ & $\mathrm{~S}$ & PEG 4000 & 31.0 & $\%(w / v)$ \\
\hline $\mathrm{C} 4$ & 4 & Citrate $\mathrm{pH} 5.5$ & 0.15 & $M$ & $\mathrm{~F}$ & Sodium iodide & 0.120 & $M$ & $\mathrm{~T}$ & Lithium sulfate & 1.0 & $M$ \\
\hline C5 & 5 & MES pH 6.0 & 0.15 & $M$ & $\mathrm{G}$ & Propanediol & 6.000 & $\%(v / v)$ & $\mathrm{U}$ & PEG MME 5000 & 26.0 & $\%(w / v)$ \\
\hline C6 & 6 & Cacodylate $\mathrm{pH} 6.5$ & 0.15 & $M$ & $\mathrm{H}$ & Calcium chloride & 0.060 & M & $\mathrm{V}$ & Glycerol & 47.0 & $\%(w / v)$ \\
\hline C7 & 7 & MOPS pH 7.0 & 0.15 & $M$ & I & Ethylene glycol & 6.000 & $\%(v / v)$ & W & Ammonium sulfate & 1.8 & $M$ \\
\hline $\mathrm{C} 8$ & 8 & HEPES pH 7.5 & 0.15 & $M$ & $\mathrm{~J}$ & Sodium potassium tartrate & 0.060 & $M$ & $\mathrm{X}$ & PEG 8000 & 26.0 & $\%(w / v)$ \\
\hline C9 & 9 & Tris pH 8.0 & 0.15 & $M$ & $\mathrm{~K}$ & MPD & 6.000 & $\%(v / v)$ & M & Phosphate & 0.8 & $M$ \\
\hline $\mathrm{C} 10$ & 10 & TAPS pH 8.5 & 0.15 & $M$ & $\mathrm{~L}$ & 2-Butanol & 6.000 & $\%(v / v)$ & $\mathrm{N}$ & PEG MME 550 & 31.00 & $\%(v / v)$ \\
\hline C11 & 11 & AMPD/Tris pH 9.0 & 0.15 & $M$ & A & Potassium bromide & 0.120 & $M$ & $\mathrm{O}$ & Ammonium nitrate & 2.6 & $M$ \\
\hline $\mathrm{C} 12$ & 12 & CAPSO pH 9.5 & 0.15 & $M$ & B & PEG 300 & 6.000 & $\%(v / v)$ & $\mathrm{P}$ & PEG 20000 & 13.0 & $\%(w / v)$ \\
\hline D1 & 1 & Formate $\mathrm{pH} 4.0$ & 0.15 & $M$ & $\mathrm{~J}$ & & & & $\mathrm{~S}$ & PEG 4000 & 35.0 & $\%(w / v)$ \\
\hline D2 & 2 & Acetate $\mathrm{pH} 4.5$ & 0.15 & $M$ & $\mathrm{~K}$ & MPD & 5.000 & $\%(v / v)$ & $\mathrm{T}$ & Lithium sulfate & 1.1 & $M$ \\
\hline D3 & 3 & Malate pH 5.0 & 0.15 & $M$ & $\mathrm{~L}$ & 2-Butanol & 5.000 & $\%(v / v)$ & $\mathrm{U}$ & PEG MME 5000 & 38.00 & $\%(v / v)$ \\
\hline D4 & 4 & Citrate $\mathrm{pH} 5.5$ & 0.15 & $M$ & A & Potassium bromide & 0.100 & $M$ & $\mathrm{~V}$ & Glycerol & 52.0 & $\%(w / v)$ \\
\hline D5 & 5 & MES pH 6.0 & 0.15 & $M$ & B & PEG 300 & 5.000 & $\%(v / v)$ & W & Ammonium sulfate & 2.0 & $M$ \\
\hline D6 & 6 & Cacodylate $\mathrm{pH} 6.5$ & 0.15 & $M$ & $\mathrm{C}$ & Magnesium sulfate & 0.100 & $M$ & $\mathrm{X}$ & PEG 8000 & 29.0 & $\%(w / v)$ \\
\hline D7 & 7 & MOPS pH 7.0 & 0.15 & $M$ & $\mathrm{D}$ & Sodium fluoride & 0.020 & $M$ & M & Phosphate & 0.9 & $M$ \\
\hline D8 & 8 & HEPES pH 7.5 & 0.15 & $M$ & E & Potassium thiocyanate & 0.050 & $M$ & $\mathrm{~N}$ & PEG MME 550 & 34.00 & $\%(v / v)$ \\
\hline D9 & 9 & Tris pH 8.0 & 0.15 & $M$ & $\mathrm{~F}$ & Sodium iodide & 0.100 & $M$ & $\mathrm{O}$ & Ammonium nitrate & 2.9 & $M$ \\
\hline D10 & 10 & TAPS pH 8.5 & 0.15 & $M$ & G & Propanediol & 5.000 & $\%(v / v)$ & $\mathrm{P}$ & PEG 20000 & 15.0 & $\%(w / v)$ \\
\hline D11 & 11 & & & & $\mathrm{H}$ & Calcium chloride & 0.050 & $M$ & $\mathrm{Q}$ & PEG 1000 & 43.0 & $\%(w / v)$ \\
\hline D12 & 12 & CAPSO pH 9.5 & 0.15 & $M$ & I & Ethylene glycol & 5.000 & $\%(v / v)$ & $\mathrm{R}$ & Sodium chloride & 2.3 & $M$ \\
\hline E1 & 1 & Formate $\mathrm{pH} 4.0$ & 0.15 & $M$ & $\mathrm{E}$ & Potassium thiocyanate & 0.040 & $M$ & $\mathrm{U}$ & PEG MME 5000 & 32.0 & $\%(w / v)$ \\
\hline E2 & 2 & Acetate $\mathrm{pH} 4.5$ & 0.15 & $M$ & $\mathrm{~F}$ & Sodium iodide & 0.080 & $M$ & $\mathrm{~V}$ & Glycerol & 57.0 & $\%(w / v)$ \\
\hline E3 & 3 & Malate pH 5.0 & 0.15 & $M$ & $\mathrm{G}$ & Propanediol & 4.000 & $\%(v / v)$ & W & Ammonium sulfate & 2.2 & $M$ \\
\hline E4 & 4 & Citrate pH 5.5 & 0.15 & $M$ & $\mathrm{H}$ & & & & $\mathrm{X}$ & PEG 8000 & 32.0 & $\%(w / v)$ \\
\hline E5 & 5 & MES pH 6.0 & 0.15 & $M$ & I & Ethylene glycol & 4.000 & $\%(v / v)$ & $\mathrm{M}$ & Phosphate & 0.9 & M \\
\hline E6 & 6 & Cacodylate pH 6.5 & 0.15 & $M$ & $\mathrm{~J}$ & Sodium potassium tartrate & 0.040 & $M$ & $\mathrm{~N}$ & PEG MME 550 & 38.0 & $\%(v / v)$ \\
\hline E7 & 7 & MOPS pH 7.0 & 0.15 & $M$ & $\mathrm{~K}$ & MPD & 4.000 & $\%(v / v)$ & $\mathrm{O}$ & Ammonium nitrate & 3.1 & $M$ \\
\hline E8 & 8 & HEPES pH 7.5 & 0.15 & $M$ & $\mathrm{~L}$ & 2-Butanol & 4.000 & $\%(v / v)$ & $\mathrm{P}$ & PEG 20000 & 16.0 & $\%(w / v)$ \\
\hline E9 & 9 & Tris $\mathrm{pH} 8.0$ & 0.15 & $M$ & A & Potassium bromide & 0.080 & $M$ & Q & PEG 1000 & 48.0 & $\%(w / v)$ \\
\hline E10 & 10 & TAPS pH 8.5 & 0.15 & $M$ & B & PEG 300 & 4.000 & $\%(v / v)$ & $\mathrm{R}$ & Sodium chloride & 2.5 & $M$ \\
\hline E11 & 11 & AMPD/Tris pH 9.0 & 0.15 & $M$ & $\mathrm{C}$ & Magnesium sulfate & 0.080 & $M$ & $\mathrm{~S}$ & PEG 4000 & 38.0 & $\%(w / v)$ \\
\hline E12 & 12 & CAPSO pH 9.5 & 0.15 & $M$ & $\mathrm{D}$ & & & & $\mathrm{T}$ & Lithium sulfate & 1.3 & $M$ \\
\hline F1 & 1 & Formate $\mathrm{pH} 4.0$ & 0.15 & $M$ & $\mathrm{~L}$ & 2-Butanol & 3.000 & $\%(v / v)$ & $\mathrm{W}$ & Ammonium sulfate & 2.4 & $M$ \\
\hline $\mathrm{F} 2$ & 2 & Acetate $\mathrm{pH} 4.5$ & 0.15 & $M$ & A & Potassium bromide & 0.060 & $M$ & $\mathrm{X}$ & PEG 8000 & 35.0 & $\%(w / v)$ \\
\hline F3 & 3 & Malate pH 5.0 & 0.15 & $M$ & B & PEG 300 & 3.000 & $\%(v / v)$ & M & Phosphate & 1.0 & $M$ \\
\hline F4 & 4 & & & & $\mathrm{C}$ & Magnesium sulfate & 0.06 & $M$ & $\mathrm{~N}$ & PEG MME 550 & 42.00 & $\%(v / v)$ \\
\hline F5 & 5 & MES pH 6.0 & 0.15 & $M$ & $\mathrm{D}$ & Sodium fluoride & 0.012 & $M$ & $\mathrm{O}$ & Ammonium nitrate & 3.4 & $M$ \\
\hline
\end{tabular}


Table 1 (continued)

\begin{tabular}{|c|c|c|c|c|c|c|c|c|c|c|c|c|}
\hline \multirow[b]{2}{*}{ Well } & \multicolumn{4}{|c|}{ Set 1} & \multicolumn{4}{|c|}{ Set 2} & \multicolumn{4}{|c|}{ Set 3} \\
\hline & ID & Name & Conc. & Unit & ID & Name & Conc. & Unit & ID & Name & Conc. & Unit \\
\hline F6 & 6 & Cacodylate $\mathrm{pH} 6.5$ & 0.15 & $M$ & $\mathrm{E}$ & Potassium thiocyanate & 0.030 & $M$ & $\mathrm{P}$ & PEG 20000 & 18.0 & $\%(w / v)$ \\
\hline F7 & 7 & MOPS pH 7.0 & 0.15 & $M$ & $\mathrm{~F}$ & Sodium iodide & 0.060 & $M$ & Q & PEG 1000 & 52.0 & $\%(w / v)$ \\
\hline F8 & 8 & HEPES pH 7.5 & 0.15 & $M$ & G & Propanediol & 3.000 & $\%(v / v)$ & $\mathrm{R}$ & Sodium chloride & 2.7 & $M$ \\
\hline F9 & 9 & Tris $\mathrm{pH} 8.0$ & 0.15 & $M$ & $\mathrm{H}$ & Calcium chloride & 0.030 & $M$ & $\mathrm{~S}$ & PEG 4000 & 42.0 & $\%(w / v)$ \\
\hline F10 & 10 & TAPS pH 8.5 & 0.15 & $M$ & I & Ethylene glycol & 3.000 & $\%(v / v)$ & $\mathrm{T}$ & Lithium sulfate & 1.4 & $M$ \\
\hline F11 & 11 & AMPD/Tris pH 9.0 & 0.15 & $M$ & $\mathrm{~J}$ & Sodium potassium tartrate & 0.030 & $M$ & $\mathrm{U}$ & PEG MME 5000 & 35.0 & $\%(w / v)$ \\
\hline $\mathrm{F} 12$ & 12 & CAPSO pH 9.5 & 0.15 & $M$ & $\mathrm{~K}$ & MPD & 3.000 & $\%(v / v)$ & $\mathrm{V}$ & Glycerol & 62.0 & $\%(w / v)$ \\
\hline G1 & 1 & Formate $\mathrm{pH} 4.0$ & 0.15 & $M$ & G & Propanediol & 2.000 & $\%(v / v)$ & M & Phosphate & 1.1 & $M$ \\
\hline G2 & 2 & Acetate $\mathrm{pH} 4.5$ & 0.15 & $M$ & $\mathrm{H}$ & & & & $\mathrm{N}$ & PEG MME 550 & 45.00 & $\%(v / v)$ \\
\hline G3 & 3 & Malate pH 5.0 & 0.15 & $M$ & I & Ethylene glycol & 2.000 & $\%(v / v)$ & $\mathrm{O}$ & Ammonium nitrate & 3.7 & $M$ \\
\hline G4 & 4 & Citrate $\mathrm{pH} 5.5$ & 0.15 & $M$ & $\mathrm{~J}$ & Sodium potassium tartrate & 0.020 & $M$ & $\mathrm{P}$ & PEG 20000 & 19.0 & $\%(w / v)$ \\
\hline G5 & 5 & MES pH 6.0 & 0.15 & $M$ & $\mathrm{~K}$ & MPD & 2.000 & $\%(v / v)$ & Q & PEG 1000 & 56.0 & $\%(w / v)$ \\
\hline G6 & 6 & Cacodylate $\mathrm{pH} 6.5$ & 0.15 & $M$ & $\mathrm{~L}$ & 2-Butanol & 2.000 & $\%(v / v)$ & $\mathrm{R}$ & Sodium chloride & 3.0 & $M$ \\
\hline G7 & 7 & MOPS pH 7.0 & 0.15 & $M$ & A & Potassium bromide & 0.040 & $M$ & $\mathrm{~S}$ & PEG 4000 & 45.0 & $\%(w / v)$ \\
\hline G8 & 8 & HEPES pH 7.5 & 0.15 & $M$ & $\mathrm{~B}$ & PEG 300 & 2.000 & $\%(v / v)$ & $\mathrm{T}$ & Lithium sulfate & 1.5 & $M$ \\
\hline G9 & 9 & Tris $\mathrm{pH} 8.0$ & 0.15 & $M$ & $\mathrm{C}$ & Magnesium sulfate & 0.040 & $M$ & $\mathrm{U}$ & PEG MME 5000 & 38.0 & $\%(w / v)$ \\
\hline G10 & 10 & TAPS pH 8.5 & 0.15 & $M$ & $\mathrm{D}$ & Sodium fluoride & 0.008 & $M$ & $\mathrm{~V}$ & Glycerol & 67.0 & $\%(w / v)$ \\
\hline G11 & 11 & AMPD/Tris pH 9.0 & 0.15 & $M$ & $\mathrm{E}$ & Potassium thiocyanate & 0.020 & $M$ & $\mathrm{~W}$ & Ammonium sulfate & 2.6 & $M$ \\
\hline G12 & 12 & CAPSO pH 9.5 & 0.15 & $M$ & $\mathrm{~F}$ & Sodium iodide & 0.040 & $M$ & $\mathrm{X}$ & PEG 8000 & 38.0 & $\%(w / v)$ \\
\hline H1 & 1 & Formate $\mathrm{pH} 4.0$ & 0.15 & $M$ & $\mathrm{~B}$ & PEG 300 & 1.000 & $\%(v / v)$ & $\mathrm{O}$ & Ammonium nitrate & 4.0 & $M$ \\
\hline $\mathrm{H} 2$ & 2 & Acetate $\mathrm{pH} 4.5$ & 0.15 & $M$ & $\mathrm{C}$ & Magnesium sulfate & 0.020 & $M$ & $\mathrm{P}$ & PEG 20000 & 20.0 & $\%(w / v)$ \\
\hline H3 & 3 & Malate pH 5.0 & 0.15 & $M$ & $\mathrm{D}$ & Sodium fluoride & 0.004 & $M$ & Q & PEG 1000 & 60.0 & $\%(w / v)$ \\
\hline $\mathrm{H} 4$ & 4 & Citrate $\mathrm{pH} 5.5$ & 0.15 & $M$ & $\mathrm{E}$ & Potassium thiocyanate & 0.010 & $M$ & $\mathrm{R}$ & Sodium chloride & 3.2 & $M$ \\
\hline H5 & 5 & MES pH 6.0 & 0.15 & $M$ & $\mathrm{~F}$ & Sodium iodide & 0.020 & $M$ & $\mathrm{~S}$ & PEG 4000 & 48.0 & $\%(w / v)$ \\
\hline H6 & 6 & Cacodylate pH 6.5 & 0.15 & $M$ & G & Propanediol & 1.000 & $\%(v / v)$ & $\mathrm{T}$ & Lithium sulfate & 1.6 & $M$ \\
\hline $\mathrm{H} 7$ & 7 & MOPS pH 7.0 & 0.15 & $M$ & $\mathrm{H}$ & Calcium chloride & 0.010 & $M$ & $\mathrm{U}$ & PEG MME 5000 & 40.0 & $\%(w / v)$ \\
\hline $\mathrm{H} 8$ & 8 & HEPES pH 7.5 & 0.15 & $M$ & I & Ethylene glycol & 1.000 & $\%(v / v)$ & $\mathrm{V}$ & Glycerol & 72.0 & $\%(w / v)$ \\
\hline H9 & 9 & Tris $\mathrm{pH} 8.0$ & 0.15 & $M$ & $\mathrm{~J}$ & Sodium potassium tartrate & 0.010 & $M$ & $\mathrm{~W}$ & Ammonium sulfate & 2.8 & $M$ \\
\hline H10 & 10 & TAPS pH 8.5 & 0.15 & $M$ & $\mathrm{~K}$ & MPD & 1.000 & $\%(v / v)$ & $\mathrm{X}$ & PEG 8000 & 40.0 & $\%(w / v)$ \\
\hline H11 & 11 & AMPD/Tris pH 9.0 & 0.15 & $M$ & $\mathrm{~L}$ & 2-Butanol & 1.000 & $\%(v / v)$ & M & Phosphate & 1.2 & $M$ \\
\hline H12 & 12 & CAPSO pH 9.5 & 0.15 & $M$ & A & Potassium bromide & 0.020 & $M$ & $\mathrm{~N}$ & PEG MME 550 & 48.00 & $\%(v / v)$ \\
\hline
\end{tabular}

Table 2

Details of the samples used with the Pi minimal screen (Table 1) and number of crystallization hits.

TEN, buffer consisting of $20 \mathrm{~m} M$ 2-amino-2-(hydroxymethyl)-1,3-propanediol (Tris), $1 \mathrm{~m} M$ ethylenediaminetetraacetic acid (EDTA), $1 \mathrm{~m} M$ sodium azide and $200 \mathrm{~m} M$ sodium chloride.

\begin{tabular}{llrllr}
\hline Protein & $\begin{array}{l}\text { Concentration } \\
\left(\mathrm{mg} \mathrm{ml}^{-1}\right)\end{array}$ & $\begin{array}{l}\text { MW } \\
(\mathrm{kDa})\end{array}$ & Source and code & Buffer/preparation & Hits \\
\hline Lysozyme & 10.0 & 14.4 & Sigma L6876 & Deionized water & 56 \\
Concanavalin A & 7.0 & 26.5 & Sigma L7647 & TEN pH 8.5 & 16 \\
Glucose isomerase & 33.0 & 43.0 & Hampton HR7-102 & See product user guide & 11 \\
Xylanase & 36.0 & 21.0 & Hampton HR7-106 & See product user guide & 8 \\
Ferritin & $50-150$ & 440.0 & Fluka 96701 & As supplied by the manufacturer & 8 \\
Catalase & 12.6 & 62.5 & Sigma C3155 & Deionized water & 6 \\
Citrate synthase & 10.0 & 49.0 & Sigma C3260 & TEN pH 8.5 & 5 \\
Lipase B & 25.0 & 35.0 & Hampton HR7-099 & Deionized water & 4 \\
Ribonuclease A & 30.0 & 13.7 & Sigma R5503 & Deionized water & 1 \\
Thaumatin & 30.0 & 22.0 & Sigma T7638 & Deionized water & 1 \\
Sum & & & & & 116 \\
\hline
\end{tabular}

\section{Table 3}

'Hits per condition' observed and corresponding results expected with the binomial distribution (Pi minimal screen).

\begin{tabular}{lll}
\hline Hits/condition & Observed & Expected \\
\hline 0 & 21 & 26.5 \\
1 & 45 & 36.4 \\
2 & 20 & 22.5 \\
3 or more & 10 & 10.6 \\
Sum & 96 & 96 \\
\hline
\end{tabular}

synchrotron light source (microfocus beamline I24 equipped with a Pilatus $6 \mathrm{M}$ detector).

\section{Results}

There were 116 crystallization hits overall for the experiments with the $\mathrm{Pi}$ minimal screen (Table 2). Some conditions produced hits for several samples (Table 3).

The Pi-PEG screen yielded crystals that diffracted to $3.0 \AA$ resolution for $\mathrm{A}_{2 \mathrm{~A}}$ R-GL31 with bound agonist. Fig. 3 shows the crystals of $\mathrm{A}_{2 \mathrm{~A}} \mathrm{R}-\mathrm{GL} 31$ obtained in well E9 [50 $\mathrm{m} M$ Tris- $\mathrm{HCl}$ $\mathrm{pH} 7.6,9.6 \%(v / v)$ PEG 200, 22.9\%(v/v) PEG 300] and an example of the corresponding diffraction pattern (no cryoprotectant was required).

\section{Discussion}

\subsection{Pi sampling}

In order to understand the rationale behind the modular arithmetic 
Table 4

Final formulation of the Pi-PEG screen.

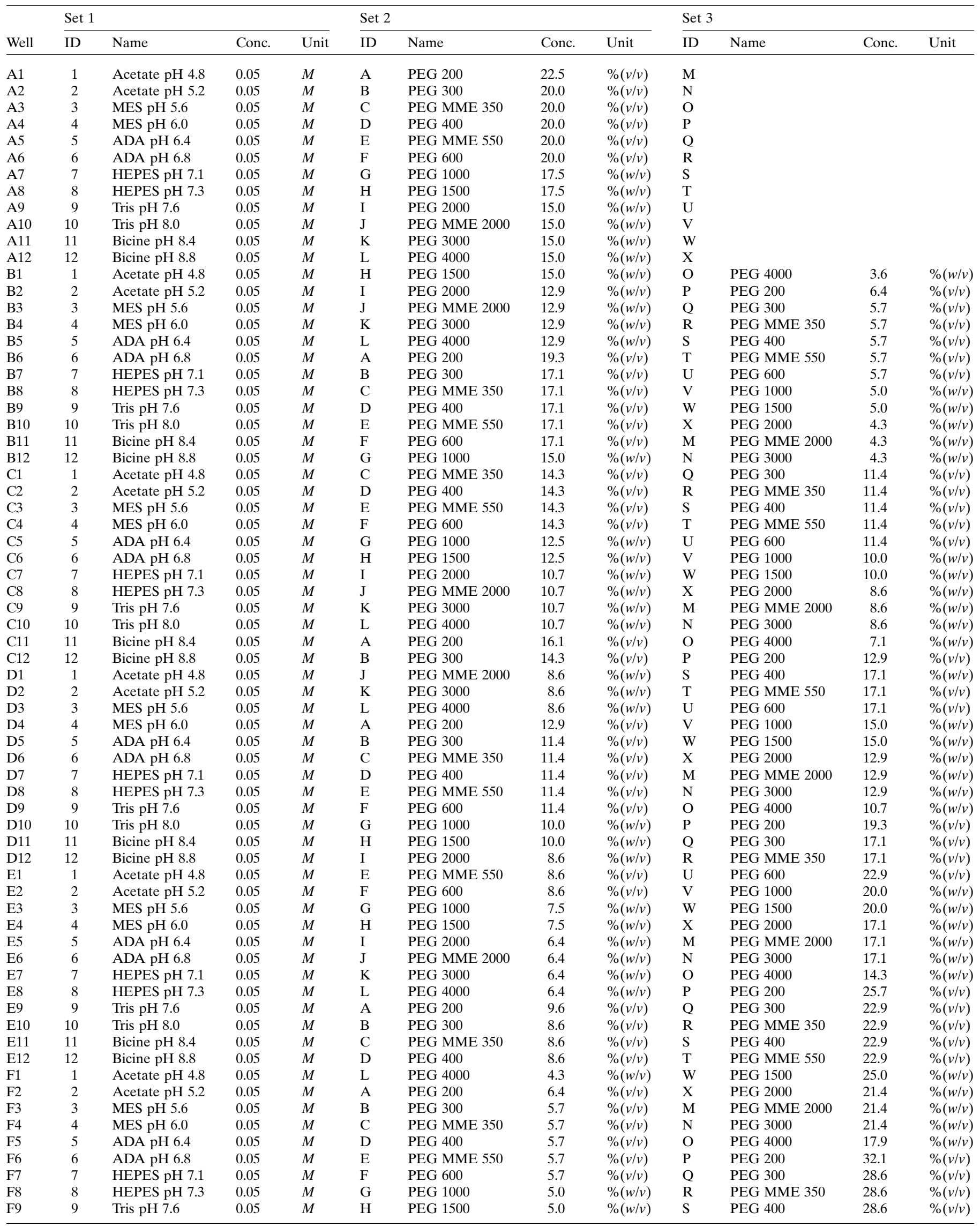


Table 4 (continued)

\begin{tabular}{|c|c|c|c|c|c|c|c|c|c|c|c|c|}
\hline \multirow[b]{2}{*}{ Well } & \multicolumn{4}{|c|}{ Set 1} & \multicolumn{4}{|c|}{ Set 2} & \multicolumn{4}{|c|}{ Set 3} \\
\hline & ID & Name & Conc. & Unit & ID & Name & Conc. & Unit & ID & Name & Conc. & Unit \\
\hline F10 & 10 & Tris $\mathrm{pH} 8.0$ & 0.05 & $M$ & I & PEG 2000 & 4.3 & $\%(w / v)$ & $\mathrm{T}$ & PEG MME 550 & 28.6 & $\%(v / v)$ \\
\hline F11 & 11 & Bicine $\mathrm{pH} 8.4$ & 0.05 & $M$ & $\mathrm{~J}$ & PEG MME 2000 & 4.3 & $\%(w / v)$ & $\mathrm{U}$ & PEG 600 & 28.6 & $\%(v / v)$ \\
\hline F12 & 12 & Bicine $\mathrm{pH} 8.8$ & 0.05 & $M$ & $\mathrm{~K}$ & PEG 3000 & 4.3 & $\%(w / v)$ & $\mathrm{V}$ & PEG 1000 & 25.0 & $\%(w / v)$ \\
\hline G1 & 1 & Acetate $\mathrm{pH} 4.8$ & 0.05 & $M$ & $\mathrm{G}$ & PEG 1000 & 2.5 & $\%(w / v)$ & M & PEG MME 2000 & 25.7 & $\%(w / v)$ \\
\hline G2 & 2 & Acetate pH 5.2 & 0.05 & $M$ & $\mathrm{H}$ & PEG 1500 & 2.5 & $\%(w / v)$ & $\mathrm{N}$ & PEG 3000 & 25.7 & $\%(w / v)$ \\
\hline G3 & 3 & MES pH 5.6 & 0.05 & $M$ & I & PEG 2000 & 2.1 & $\%(w / v)$ & $\mathrm{O}$ & PEG 4000 & 21.4 & $\%(w / v)$ \\
\hline G4 & 4 & MES pH 6.0 & 0.05 & $M$ & $\mathbf{J}$ & PEG MME 2000 & 2.1 & $\%(w / v)$ & $\mathrm{P}$ & PEG 200 & 38.6 & $\%(v / v)$ \\
\hline G5 & 5 & ADA pH 6.4 & 0.05 & $M$ & $\mathrm{~K}$ & PEG 3000 & 2.1 & $\%(w / v)$ & Q & PEG 300 & 34.3 & $\%(v / v)$ \\
\hline G6 & 6 & ADA pH 6.8 & 0.05 & $M$ & $\mathrm{~L}$ & PEG 4000 & 2.1 & $\%(w / v)$ & $\mathrm{R}$ & PEG MME 350 & 34.3 & $\%(v / v)$ \\
\hline G7 & 7 & HEPES pH 7.1 & 0.05 & $M$ & A & PEG 200 & 3.2 & $\%(v / v)$ & $\mathrm{S}$ & PEG 400 & 34.3 & $\%(v / v)$ \\
\hline G8 & 8 & HEPES pH 7.3 & 0.05 & $M$ & B & PEG 300 & 2.9 & $\%(v / v)$ & $\mathrm{T}$ & PEG MME 550 & 34.3 & $\%(v / v)$ \\
\hline G9 & 9 & Tris $\mathrm{pH} 7.6$ & 0.05 & $M$ & $\mathrm{C}$ & PEG MME 350 & 2.9 & $\%(v / v)$ & $\mathrm{U}$ & PEG 600 & 34.3 & $\%(v / v)$ \\
\hline G10 & 10 & Tris $\mathrm{pH} 8.0$ & 0.05 & $M$ & $\mathrm{D}$ & PEG 400 & 2.9 & $\%(v / v)$ & $\mathrm{V}$ & PEG 1000 & 30.0 & $\%(w / v)$ \\
\hline G11 & 11 & Bicine $\mathrm{pH} 8.4$ & 0.05 & $M$ & $\mathrm{E}$ & PEG MME 550 & 2.9 & $\%(v / v)$ & $\mathrm{W}$ & PEG 1500 & 30.0 & $\%(w / v)$ \\
\hline G12 & 12 & Bicine $\mathrm{pH} 8.8$ & 0.05 & $M$ & $\mathrm{~F}$ & PEG 600 & 2.9 & $\%(v / v)$ & $\mathrm{X}$ & PEG 2000 & 25.7 & $\%(w / v)$ \\
\hline H1 & 1 & Acetate $\mathrm{pH} 4.8$ & 0.05 & $M$ & B & & & & $\mathrm{O}$ & PEG 4000 & 25.0 & $\%(w / v)$ \\
\hline $\mathrm{H} 2$ & 2 & Acetate pH 5.2 & 0.05 & $M$ & $\mathrm{C}$ & & & & $\mathrm{P}$ & PEG 200 & 45.0 & $\%(v / v)$ \\
\hline $\mathrm{H} 3$ & 3 & MES pH 5.6 & 0.05 & $M$ & $\mathrm{D}$ & & & & Q & PEG 300 & 40.0 & $\%(v / v)$ \\
\hline $\mathrm{H} 4$ & 4 & MES pH 6.0 & 0.05 & $M$ & $\mathrm{E}$ & & & & $\mathrm{R}$ & PEG MME 350 & 40.0 & $\%(v / v)$ \\
\hline H5 & 5 & ADA pH 6.4 & 0.05 & $M$ & $\mathrm{~F}$ & & & & $\mathrm{~S}$ & PEG 400 & 40.0 & $\%(v / v)$ \\
\hline H6 & 6 & ADA pH 6.8 & 0.05 & $M$ & $\mathrm{G}$ & & & & $\mathrm{T}$ & PEG MME 550 & 40.0 & $\%(v / v)$ \\
\hline $\mathrm{H} 7$ & 7 & HEPES pH 7.1 & 0.05 & $M$ & $\mathrm{H}$ & & & & $\mathrm{U}$ & PEG 600 & 40.0 & $\%(v / v)$ \\
\hline $\mathrm{H} 8$ & 8 & HEPES pH 7.3 & 0.05 & $M$ & $\mathrm{I}$ & & & & $\mathrm{V}$ & PEG 1000 & 35.0 & $\%(w / v)$ \\
\hline H9 & 9 & Tris $\mathrm{pH} 7.6$ & 0.05 & $M$ & $\mathrm{~J}$ & & & & $\mathrm{~W}$ & PEG 1500 & 35.0 & $\%(w / v)$ \\
\hline H10 & 10 & Tris $\mathrm{pH} 8.0$ & 0.05 & $M$ & $\mathrm{~K}$ & & & & $\mathrm{X}$ & PEG 2000 & 30.0 & $\%(w / v)$ \\
\hline H11 & 11 & Bicine $\mathrm{pH} 8.4$ & 0.05 & $M$ & $\mathrm{~L}$ & & & & $\mathrm{M}$ & PEG MME 2000 & 30.0 & $\%(w / v)$ \\
\hline H12 & 12 & Bicine $\mathrm{pH} 8.8$ & 0.05 & $M$ & A & & & & $\mathrm{N}$ & PEG 3000 & 30.0 & $\%(w / v)$ \\
\hline
\end{tabular}
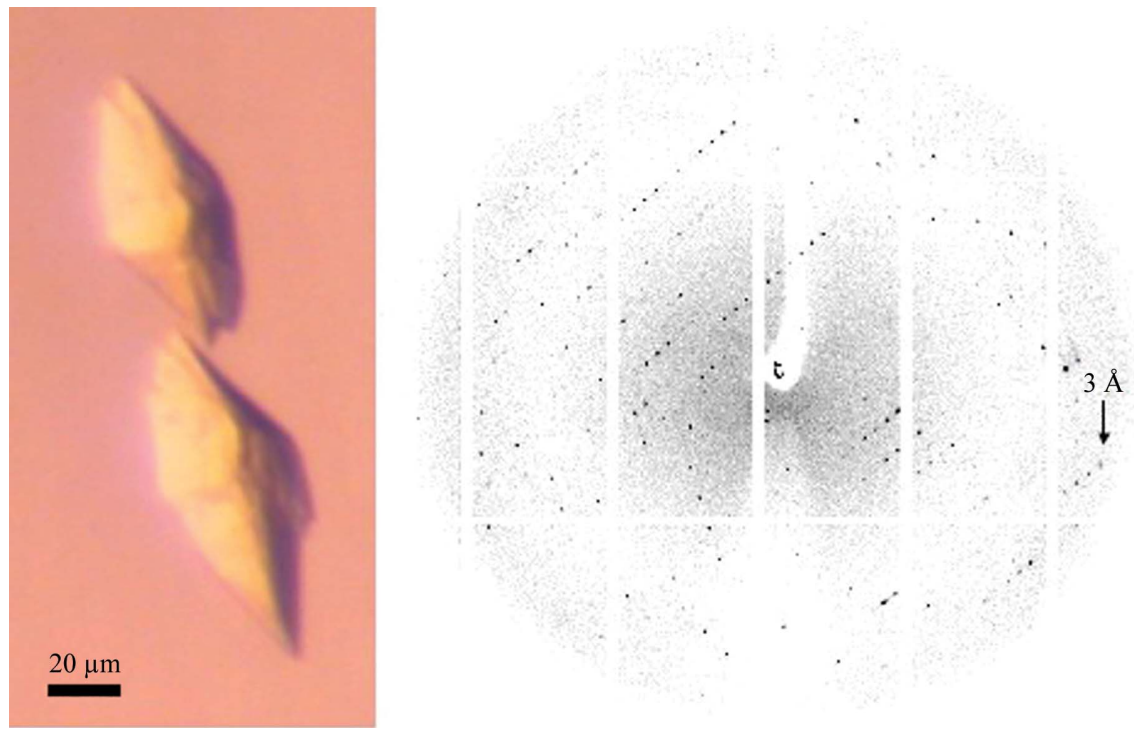

Figure 3

Crystals of $\mathrm{A}_{2 \mathrm{~A}} \mathrm{R}$-GL31 obtained with the Pi-PEG screen (Table 4) and an example of a corresponding diffraction pattern.

employed for the Pi sampling, it may help to imagine, on a $12 \mathrm{~h}$ clock, a series of events occurring every $5 \mathrm{~h}$. The first event is at noon, the second at $5 \mathrm{pm}$, then $10 \mathrm{pm}$, then $3 \mathrm{am}$ etc. Eventually, there is a succession of 12 events occurring at different hours, with as much time as possible in between each event. If we now look at combinations of three components, there are originally $12^{3}$ or 1728 possibilities. Pi Sampler generates 96 of these combinations that correspond to conditions that are distant in properties. The variety between conditions is then accentuated using a number of different concentrations of solutions (Fig. 2). If the first and second sets of solutions are ordered according to physico-chemical properties, the generated screen will be an incomplete factorial sampling of interactions between chemicals with these properties. If the chemicals selected have completely different natures, they can be arranged randomly (see \$2.3). The ordering of the third set of solutions can be used to avoid obvious chemical incompatibilities (e.g. mixing phosphate and magnesium salts). It is also possible to design simpler screens with only two sets of stock solutions.

\subsection{The Pi minimal screen}

In order to check the homogeneity of the hits across the screen with the ten samples, we compared the results obtained with what would be expected if each condition had the same probability of hits overall (Table 3). This can be approximated by a binomial distribution. The probability of success for the binomial distribution is the observed probability for ten attempts: $116 /$ $(10 \times 96)=0.12083$. The $\chi^{2}$ statistic for the data is 3.48 . This can be compared with the quantiles of a $\chi^{2}$ distribution with two degrees of freedom, which gives a $p$ value of 0.18 (calculations not shown). This $\chi^{2}$ test indicates that no 
conditions are obvious outliers with regard to success or failure. There are, however, a multitude of possible biases implied when proceeding with crystallization experiments (which would be even more accentuated with the use of novel samples); hence, any statistical analysis should be taken with precaution. Nonetheless, it is interesting to see that the analysis of the distribution is in accordance with the original approach based on balanced randomization (Carter \& Carter, 1979; Rupp, 2003).

In addition, the conditions of the Pi minimal screen show no identities to the extensive list of conditions (7230) from commercial screens stored in the 'PICKScreens' database (Hedderich et al., 2011).

\subsection{The Pi-PEG screen}

The extent of effects on crystallization for precipitants such as PEGs is correlated with their concentrations (McPherson, 1976) and molecular weights (Forsythe et al., 2002). The PiPEG screen covers a wide range of parameters (kinetics of equilibrium, protein stabilization etc.). In addition, the concentrations of the two different PEGs in a condition can be adjusted for condition optimization (Stock et al., 2005) and for crystal cryoprotection (Berejnov et al., 2006). Furthermore, the PICKScreens database shows that the Pi-PEG screen is unique (as for the Pi minimal screen; see $\S 4.2$ ).

Samples of $A_{2 A}$ R-GL31 purified in a number of different detergents rarely crystallized in commercially available screens used at the LMB (Stock et al., 2005) and when they did the crystal quality was not sufficient for structure determination. The first quality crystals were recently obtained using the Pi-PEG screen.

\section{Conclusions}

We have demonstrated that the Pi sampling is a methodical and flexible approach to initial screening for macromolecular crystallization. Two unique screens produced de novo have resulted from this strategy. The Pi minimal screen potentially has an ideal formulation for crystallization of novel soluble protein samples. The Pi-PEG screen is a tailor-made screen for GPCRs and potentially other membrane proteins generated by biasing the formulation towards components known to be essential.

Further screens can be formulated with the Pi Sampler on a day-to-day basis in order to test chemicals and techniques, with the aim of increasing the yield of quality crystals. Also, new crystallization techniques are constantly emerging for macromolecular targets such as membrane proteins and hence formulations with special considerations are required: one may want to formulate screens compatible with the lipidic cubic phase (LCP) concept (Landau \& Rosenbusch, 1996) or make extensive use of detergents (Koszelak-Rosenblum et al., 2009).
In order for laboratories to be able to handle many $\mathrm{Pi}$ screen formulations and the flow of resulting data, we are working on the integration of Pi Sampler into the 'xtalPiMS' Laboratory Information Management System (LIMS; Morris et al., 2011; see http://www.pims-lims.org).

Thanks to Simon Byrne (Cambridge University Statistics Clinic; http://www.statslab.cam.ac.uk/clinic/) for discussions. Thanks to the LMB members Jan Löwe, John Kendrick-Jones, Christopher Aylett, Chris Tate, Jake Grimmett and Graham Lingley for various contributions. Finally, thanks to Karen Law (MRC Technology), Chris Morris (STFC, funded by CCP4) and Tanja Hedderich (Max Planck Institute). Conflicting commercial interest: we hereby state that we have a conflicting commercial interest in that MRC Technology (http://www.mrctechnology.org/) will commercialize Pi screens under an exclusive licence to Jena Bioscience (http:// www.jenabioscience.com/).

\section{References}

Berejnov, V., Husseini, N. S., Alsaied, O. A. \& Thorne, R. E. (2006). J. Appl. Cryst. 39, 244-251.

Bergfors, T. (2007). Methods Mol. Biol. 363, 131-151.

Brzozowski, A. M. \& Walton, J. (2001). J. Appl. Cryst. 34, 97-101.

Carter, C. W. \& Carter, C. W. (1979). J. Biol. Chem. 254, 12219-12223.

Clarke, O. B., Caputo, A. T., Hill, A. P., Vandenberg, J. I., Smith, B. J. \& Gulbis, J. M. (2010). Cell, 141, 1018-1029.

Cox, M. J. \& Weber, P. C. (1987). J. Appl. Cryst. 20, 366-373.

Doudna, J. A., Grosshans, C., Gooding, A. \& Kundrot, C. E. (1993). Proc. Natl Acad. Sci. USA, 90, 7829-7833.

Forsythe, E. L., Maxwell, D. L. \& Pusey, M. (2002). Acta Cryst. D58, 1601-1605.

Hedderich, T., Marcia, M., Köpke, J. \& Michel, H. (2011). Cryst. Growth Des. 11, 488-491.

Jancarik, J. \& Kim, S.-H. (1991). J. Appl. Cryst. 24, 409-411.

Kantardjieff, K. A. \& Rupp, B. (2004). Bioinformatics, 20, 2162-2168.

Kimber, M. S., Vallee, F., Houston, S., Necakov, A., Skarina, T., Evdokimova, E., Beasley, S., Christendat, D., Savchenko, A., Arrowsmith, C. H., Vedadi, M., Gerstein, M. \& Edwards, A. M. (2003). Proteins, 51, 562-568.

Koszelak-Rosenblum, M., Krol, A., Mozumdar, N., Wunsch, K., Ferin, A., Cook, E., Veatch, C. K., Nagel, R., Luft, J. R., Detitta, G. T. \& Malkowski, M. G. (2009). Protein Sci. 18, 1828-1839.

Landau, E. M. \& Rosenbusch, J. P. (1996). Proc. Natl Acad. Sci. USA, 93, 14532-14535.

Lemieux, M. J., Song, J., Kim, M. J., Huang, Y., Villa, A., Auer, M., Li, X.-D. \& Wang, D.-N. (2003). Protein Sci. 12, 2748-2756.

McPherson, A. (1976). J. Biol. Chem. 251, 6300-6303.

McPherson, A. (1990). Eur. J. Biochem. 189, 1-23.

Morris, C. et al. (2011). Acta Cryst. D67, 249-260.

Rupp, B. (2003). J. Struct. Biol. 142, 162-169.

Smith, D. E. (1958). History of Mathematics. New York: Dover Publications.

Stock, D., Perisic, O. \& Löwe, J. (2005). Prog. Biophys. Mol. Biol. 88, 311-327.

Warne, T., Serrano-Vega, M. J., Baker, J. G., Moukhametzianov, R., Edwards, P. C., Henderson, R., Leslie, A. G., Tate, C. G. \& Schertler, G. F. (2008). Nature (London), 454, 486-491.

Warne, T., Serrano-Vega, M. J., Tate, C. G. \& Schertler, G. F. (2009). Protein Expr. Purif. 65, 204-213. 